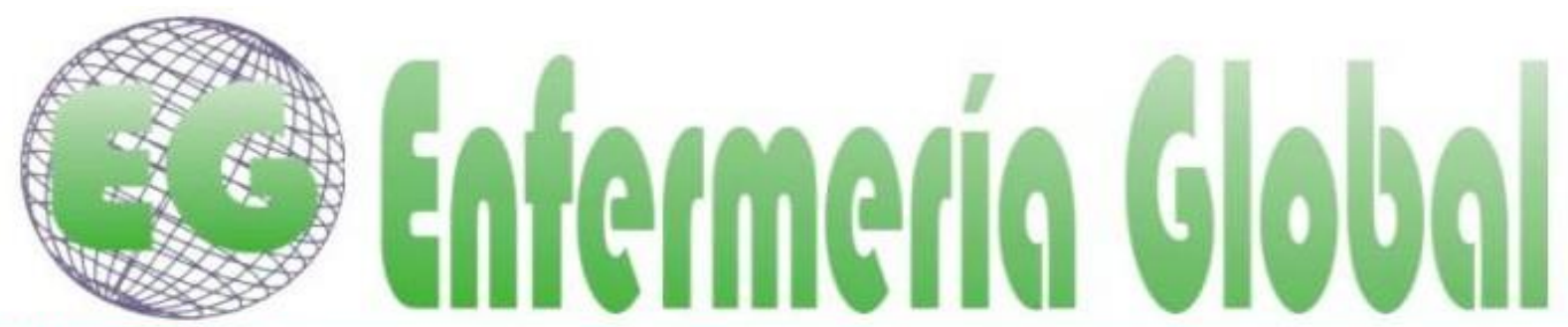

ISSN 1695-6141 Revista electrónica trimestral de Enfermería

$N^{\circ} 47$

www.um.es/eglobal/

Julio 2017

REVISIONES

\title{
Relación entre teoría y praxis en la formación de profesionales de enfermería: revisión integradora de literatura
}

Theory-praxis relationship in education of nursing professionals: an integrative literature review

\author{
Laura Elvira Piedrahita Sandoval ${ }^{1}$ \\ Ana Lucia Rosero Prado ${ }^{2}$ \\ ${ }^{1}$ Enfermera y Psicóloga. Estudiante Doctorado en Educación de la Universidad de San Buenaventura, \\ Cali. Profesor Titular, Escuela de Enfermería, Universidad del Valle. Colombia. \\ ${ }^{2}$ Doctora en Ciencias Sociales, Niñez y Juventud del Centro de Investigaciones y Estudios avanzados \\ en niñez, juventud, educación y desarrollo de la alianza CINDE y Universidad de Manizales. Colombia.
}

\section{E-mail: lauraeps1@hotmail.com}

\section{http://dx.doi.org/10.6018/eglobal.16.3.269261}

Recibido: 27/02/2016

Aceptado: 09/12/2016

\section{RESUMEN:}

El presente artículo es una revisión integradora de publicaciones de revistas indexadas inscritas en las bases de datos Scielo, MEDLINE, CINAHL, LILACS, CUIDEN, OVID, pub Med, y TESEO, entre 20002014 escritas en español, portugués e inglés.

El estudio tuvo como objetivo identificar en las producciones científicas la problematización de la relación entre teoría y praxis en la formación de los profesionales de enfermería.

Se seleccionaron 35 documentos, con base en criterios de inclusión y exclusión. Para el análisis, los estudios fueron ordenados de acuerdo a aspectos comunes como: tipo de documento, país de origen, autor, año de publicación, conceptos clave y/o definiciones. El estudio posibilitó la agrupación de los datos en unidades temáticas, emergiendo tres categorías: Estudios relacionados con los profesores y el currículo, estudios relacionados con los estudiantes y los estudios relacionados con los enfermeros asistenciales a partir de su práctica profesional.

Los resultados indican que la complejidad de la relación entre la teoría y la práctica es evidente, demostrando que no existe una transferencia del conocimiento teórico de las aulas, a la realidad de la práctica asistencial. Adicionalmente, se resalta la discreta productividad de estudios relacionados con esta temática, más teniendo en cuenta la importancia del tema y la preocupación constante que genera tanto en los profesores, estudiantes, como en los enfermeros asistenciales.

Palabras clave: enfermería; educación en enfermería; revisión integradora; docentes de enfermería; estudiantes de enfermería.

\section{ABSTRACT:}

This article presents an integrative review of studies written in Spanish, Portuguese and English, which have been published in journals indexed in the databases Scielo, MEDLINE, CINAHL, lilacs, CUIDEN, Ovid, Pub Med and TESEO, between 2000 and 2014. 
This study aimed to identify, in scientific literature, the problems of the theory-praxis relationship in the education of nursing professionals. 35 documents were selected, based on inclusion and exclusion criteria. In order to analyze them, the studies were arranged according to common aspects such as: document type, country of origin, author, year of publication, key concepts and/or definitions. This study made it possible to group the data into thematic units according to three categories: studies related to teachers and the curriculum, studies related to students, and studies related to care nurses, from their professional practice. Results indicate that complexity of the theory-praxis relationship is evident, demonstrating that there is no transfer of classroom-imparted theoretical knowledge to the actual care practice. Moreover, we highlight the inconspicuous production of studies related to this subject, considering its importance and its constant concern to teachers, students, and care nurses

Key words: Nursing; nursing education; integrative review; nursing teachers; nursing students.

\section{INTRODUCCIÓN}

La relación de la teoría con la praxis ha sido un tema constante de reflexión humana, desde los inicios de la filosofía hasta la actualidad, la cual se ha extendido a las distintas disciplinas del saber. Particularmente en enfermería la relación, coherencia o correspondencia que establecen los docentes entre el conocimiento de enfermería y la práctica institucional ha sido reconocida por diferentes autores; desde este lugar, se evidencia una tensión/ problematización tanto en los profesores, los estudiantes y los enfermeros asistenciales, como en la disciplina misma.

Etimológicamente la palabra teoría deriva del griego observar y tiene como raíz theós (dios, divinidad), por lo que su significado está vinculado con algo divino, superior, ideal, no cuestionable, digno de ser venerado y hasta temido ${ }^{(1)}$. El Diccionario de la Real Academia Española de la Lengua ${ }^{(2)}$ define la teoría como un conocimiento especulativo considerado con independencia de toda aplicación. Por su parte, la palabra praxis, deriva del griego antiguo $\pi \rho \bar{\alpha} \xi ı \varsigma$ = práctica. Ha sido traducida al latín como action y al castellano como acción. Es así como, posiblemente el primer pensador que plantea específicamente el problema de la relación entre la teoría y la praxis fue Aristóteles (384-322 a.C.), al constituir su enciclopedia de las ciencias y distinguir las ciencias propias (teóricas) de las otras disciplinas (prácticas y poiéticas). En la actualidad, autores como Bárcena ${ }^{(3)}$ manifiestan que el significado de la palabra práctica (un hacer) ha cambiado de sentido y sustenta esto retomando a Aristóteles, a partir de la distinción que hace este autor de dos formas de actividad: la praxis, (conocimiento práctico o phrónesis) y la poiesis (conocimiento productivo o tejné). Para Bárcena ${ }^{(3)}$, citado por Rivera ${ }^{(4)}$, ya no se distinguen estos dos momentos primordiales de la actividad humana, pues la producción ha acabado, de acuerdo con el autor a partir del acento que ha puesto el paradigma de la racionalidad instrumental, del saber hacer y cada vez se ha perdido el sentido de pensar lo que se hace.

En enfermería estas ideas son planteadas por Medina ${ }^{(5)}$ en España y por Benner en la literatura anglosajona. Ambos autores ponen de manifiesto la distinción entre tejné y praxis y señalan que la naturaleza de la práctica enfermera es phrónesis, al ser una disciplina que se ocupa cognoscitiva y éticamente del obrar humano. Bárcena ${ }^{(3)}$, adicionalmente señala la naturaleza práctica de la educación; afirmando que lo que verdaderamente constituye la praxis en educación es la formación. Así mismo, la autora expresa que la enfermería al igual que la educación, tienen un carácter tecnológico, pero desplegado como práctica reflexiva y ética. Autores como Medina ${ }^{(5)}$, expresan que la enseñanza de enfermería y el ejercicio profesional deben conjuntarse como una praxis y con ello trascender en un plano contextual y social más global. 
De acuerdo con Acebedo ${ }^{(6)}$, numerosos autores tales como: Bourdieu ${ }^{(7)}$, Schön ${ }^{(8)}$, Toulmin $^{(9)}$, Benner ${ }^{(10)}$ y Medina ${ }^{(11)}$, entre otros, han señalado muy claramente las diferencias que existen entre los procesos de producción y las formas de relación del saber práctico y del saber teórico, resaltando que las disciplinas prácticas, tales como la psicología, el trabajo social, la enseñanza, el derecho, la medicina o la enfermería, implican una mezcla de ciencia, tecnología y praxis, donde la praxis se desarrolla en una razón práctica, muy diferente de la razón instrumental propia del pensamiento racional y formal. Adicionalmente, señalan muy claramente las diferencias que existen entre los procesos de producción y las formas de relación del saber práctico y del saber teórico. Así mismo, autoras como Benner, critican que el único conocimiento que cuenta en el modelo racional-técnico de la práctica enfermera es el conocimiento teórico, llevando a que la práctica profesional se constituya en una aplicación instrumental de un conocimiento derivado científicamente.

López y Torres ${ }^{(12)}$, manifiestan que a partir de la década de los noventa se inicia el uso del término praxis para hablar de enfermería, como una conducta humana práctica misma que puede incluir una actividad personal, ética, estética y empírica. El considerar estos elementos en el campo de la enfermería implica dimensiones creativas, expresivas y valorativas en el conocimiento enfermero que le ayuden a un sustento científico. Por lo que hablar de praxis en enfermería, lleva a reflexionar sobre un sentido epistemológico de este término y con ello a argumentar en favor de una praxis creativa y reflexiva de enfermería reconociendo que esta se conforma en una articulación teórico-práctica, fundamentada en el conocimiento científico.

Por su parte, la teoría de enfermeria es definida por Duran de Villalobos ${ }^{(13)}$ como una comunicación de una conceptualización de algún aspecto de la realidad de la enfermería, con el propósito de describir un fenómeno, explicar las relaciones entre fenómenos, predecir las consecuencias o prescribir el cuidado de enfermería. Considerándolas adicionalmente como los depósitos donde reposan los resultados de la indagación relacionada con conceptos de enfermería, como salud, promoción de salud, comodidad, descanso, cuidado, entre otros y adicionalmente, como los depósitos de las respuestas generadas para la comprensión de los fenómenos significativos de enfermería como los procesos de recuperación, admisiones recurrentes al hospital, entre otros.

En enfermería, la problematización entre la teoría y la práctica surge a partir de la contextualización de los conceptos teoría y práctica enfermera. Desde el recorrido histórico, entre ellos se observa un origen diferenciado: la teoría tiene sus comienzos, acorde con diferentes autores; algunos la sitúan en 1852 con Nightingale ${ }^{(14)}$, mientras otros la sitúan en 1952 con Peplau. ${ }^{(15)}$ Mientras la práctica del cuidado, como actividad instintiva, tiene sus orígenes desde el comienzo de la vida ${ }^{(16)}$.

Un eje central para el avance de las disciplinas y particularmente, en este caso de la disciplina en enfermería lo constituye la teoría y la aplicación de esta a la práctica. Durán de Villalobos ${ }^{(17)}$, expresa que la enfermería siempre ha utilizado teoría para la práctica, pero históricamente han sido teorías derivadas de la disciplina médica las que primero se utilizaron como soporte de la práctica enfermera, llevando a que solo en los últimos años se haya visto un creciente interés por parte de los profesionales de enfermería por utilizar la teoría enfermera para fundamentar los marcos teóricos y discutir los hallazgos de la producción investigativa, llevando a un contexto un tanto confuso respecto a la problematización tanto de la teoría como de la praxis enfermera, que requiere una seria reflexión, particularmente desde la educación. 
Adicionalmente, se hace evidente en la revisión de literatura la débil articulación entre la teoría de enfermeria y la práctica enfermera ${ }^{(18)}$, lo que lleva a una falta de correspondencia entre la formación recibida en las instituciones educativas y las actividades realizadas por los profesionales frente a las necesidades de los servicios, generando la llamada brecha teórico-práctica, que origina una diferencia entre la formación y la práctica y se constituyen en una amenaza para el desarrollo de enfermería ${ }^{(19)}$.

Así mismo, la Red Iberoamericana de Investigación en Educación en Enfermería considera, al determinar las prioridades investigativas en educación superior en enfermería en lberoamérica, como uno de los temas prioritarios de investigación en educación en enfermería, el de la vinculación teoría-práctica en la formación del profesional de enfermería. Por su parte, Benner et al ${ }^{(20)}$ hacen un llamado hacia la necesidad de investigar la brecha existente entre la educación impartida en las escuelas de formación de profesionales de enfermería y la práctica, debido a los cambios generados por el nuevo contexto en el cual se hace necesario brindar los cuidados de salud. La autora hace un llamado al cambio desde los programas de formación de enfermeras, para cerrar la brecha entre la práctica y la educación.

En este contexto, los resultados de la presente investigación, se constituyen en un aporte para la educación en enfermería, dado que busca generar una reflexión en torno a la problemática descrita, es así como se planteó como objetivo el realizar una revisión de integración de la literatura científica, buscando fuentes documentales que aborden la problematización de la relación entre teoría y praxis en la formación de los profesionales de enfermería.

\section{METODOLOGÍA}

Estudio de enfoque cualitativo que buscó identificar en las producciones científicas la problematización de la relación entre teoría y praxis en la formación de los profesionales de enfermería. Para el alcance de este objetivo, se opto por una revisión integradora, fundamentada en los estudios de Ganong ${ }^{(21)}$, pues esta posibilita el generar nuevas perspectivas, orienta hacia la determinación de lagunas con respecto al tema estudiado y permite a partir de la síntesis de los estudios publicados generar conclusiones.

Con relación al diseño de la investigación, se optó por adoptar las seis etapas de la metodología de Ganong ${ }^{(21)}$, que son: 1) selección de la pregunta de investigación, la cual constituye la identificación del problema o cuestionamiento 2) definición de los criterios de inclusión de estudios y selección de la muestra; 3) representación de los estudios seleccionados en formato de tablas, considerando todas las características en común; 4) análisis crítico de los hallazgos, identificando diferencias y conflictos; 5) interpretación de los resultados; y 6 ) informar de forma clara la evidencia encontrada, la cual constituye la presentación de la revisión.

La selección del tema y la pregunta surge frente al problema de la relación entre la teoría y la praxis en enfermeria, de tal forma que la pregunta orientadora para este estudio fue: ¿Cuál es el estado del arte de la problematización de la relación entre teoría y praxis en la formación de los profesionales de enfermería?

La identificación y selección de los trabajos se realizó a partir del estudio bibliográfico de publicaciones indexadas en las bases de datos de la disciplina de enfermería y 
disciplinas afines al tema como educación. Se utilizaron bases de datos en español y en inglés, facilitadas por la Universidad del Valle, la Universidad de San Buenaventura y la Universidad Nacional de Colombia; tales como Scielo, MEDLINE (US NLM), CINAHL (Cumulative Index to Nursing \& Allied, lilacs, CUIDEN (Fundación Index), ovid, pub Med, y TESEO (Bases de Datos de Tesis Doctorales). Los descriptores fueron seleccionados a partir de la terminología consultada en el MESH (Nacional lybrary of Medicine's) y en los descriptores de ciencias de la salud (DECS-BIREME). Los criterios definidos para la selección de los estudios fueron: categorías de artículo: original, tesis doctoral, articulo de revista, reflexión. Estudios con resúmenes y textos completos disponibles para análisis, publicados en los idiomas español, portugués e ingles. Publicados entre los años 2000 -2014, indexados en los bancos de datos seleccionados y que contuviesen en sus títulos y/o resúmenes los siguientes descriptores: Nursing, education, teoría de enfermería, educación en enfermería, práctica profesional, enfermería práctica, docentes de enfermería y estudiantes de enfermería. El recurso utilizado en la investigación fue la expresión "término exacto" asociada a los descriptores mencionados.

Los criterios de exclusión de los estudios, fueron los documentos que no atendían a los criterios de inclusión mencionados, así como los estudios duplicados, cartas y/o disertaciones. Así como los trabajos que abordaban otras temáticas diferentes no relacionadas con el estudio.

La recolección de datos ocurrió entre los meses de agosto-noviembre de 2015. A fin de facilitar la revisión de los estudios, una vez identificados y seleccionados los textos, como instrumento de recolección, se adoptó una primera plantilla de análisis que permitió ordenar los estudios de acuerdo a aspectos comunes como: identificación de documento, país de origen, tipo de documento, autor, año de publicación, palabras clave, conceptos clave y/o definiciones. Posteriormente, se elaboró una segunda plantilla, la cual posibilitó la agrupación de los datos en unidades temáticas relacionadas con las tendencias emergentes, donde se resaltan tres actores: profesores, estudiantes y enfermeros asistenciales. De tal forma que permitieron construir tres categorías para el análisis, especificadas así: los estudios relacionados con los profesores y el currículo, los estudios relacionados con los estudiantes y los estudios relacionados con los enfermeros asistenciales a partir de su práctica profesional. Siguiendo los criterios de inclusión, fueron seleccionados 35 estudios para análisis.

\section{RESULTADOS}

De los 35 estudios analizados, 69\% (24 estudios) fueron publicados en el extranjero y $31 \%$ (11 estudios) eran nacionales. Los países de origen fueron: España 31\% (11 artículos), Brasil 17\% (6 artículos), Cuba 9\% (3 artículos), Perú, México, Chile y Venezuela con 1 artículo cada uno de ellos.

Con respecto a su categoría de publicación, 69\%, corresponden a artículos (24 artículos); $23 \%$ (8 documentos) a tesis doctorales; se clasificaron en la categoría de otros documentos los siguientes: 3\% (1) libro; 3\% (1) editorial y 3\% (1) documento institucional. Con relación a los años de publicación, la mayoría de las publicaciones se dio entre los años 2006-2010 con un 6\% (20 artículos).

Para analizar y discutir la temática de la problematización de la relación entre la teoría y la praxis en la formación de profesionales de enfermeria, siguiendo el método propuesto para el estudio, se agruparon los resultados en unidades temáticas 
relacionadas con las tendencias emergentes, especificadas así: estudios relacionados con los profesores y el currículo (18 documentos), estudios relacionados con estudiantes ( 7 documentos) y los estudios relacionados con los enfermeros asistenciales a partir de su práctica profesional (10 documentos). La tabla I muestra las tendencias emergentes del estado del arte, frente a la problematización de la relación entre teoría y praxis en la formación de profesionales de enfermería.

Tabla I: Tendencias emergentes del estado del arte, frente a la problematización de la relación entre teoría y praxis en la formación de profesionales de enfermería.

\begin{tabular}{|c|c|c|}
\hline Tendencias emergentes & $\mathrm{n}^{\circ}$ & Identificación del Estudio. \\
\hline $\begin{array}{c}\text { Estudios relacionados con los } \\
\text { profesores y con el currículo. }\end{array}$ & 18 & $\begin{array}{c}23,24,25,26,27,28,29,30,31,32,33,34,35,36,37,38, \\
39,40 .\end{array}$ \\
\hline $\begin{array}{c}\text { Estudios relacionados con los } \\
\text { estudiantes. }\end{array}$ & 7 & $41,42,43,44,45,46,47$. \\
\hline $\begin{array}{c}\text { Estudios relacionados con los } \\
\text { enfermeros asistenciales. }\end{array}$ & 10 & $48,49,50,51,52,53,14,54,55,56$. \\
\hline \multicolumn{2}{c}{ Total de estudios analizados: 35.} \\
\hline
\end{tabular}

A continuación, se describen cada una de las tendencias emergentes y los aportes que hacen al tema investigado:

Tendencia 01: estudios relacionados con los profesores y el currículo.

$51 \%$, correspondiente a 18 estudios analizados, discuten aspectos relacionados con la problematización de la teoría y la praxis por parte de los profesores, con base en otros estudios y en la literatura. Los profesores reconocen que existe un avance en el conocimiento de la disciplina, el cual no se refleja en la práctica ${ }^{22-25}$, la que se constituye en la realidad -el mundo real- y como tal se desarrolla en esta realidad. Este aspecto está aunado al desconocimiento por parte de ellos de la importancia de reconocer este desarrollo científico, pues muchos manifiestan que no conocieron en su formación el desarrollo disciplinar y han centrado su práctica en el hacer ${ }^{22}$.

Se evidencia en los trabajos revisados el llamado que hacen los autores respecto al conflicto en el cual se encuentra la enfermería, que amenaza su desarrollo como profesión y que se expresa por la falta de consenso entre los aspectos relacionados con la teoría (ciencias de enfermería) y los aspectos relacionados con la práctica enfermera (práctica profesional) ${ }^{22-25}$. Las diferentes investigaciones muestran que aunque existen importantes desarrollos de la disciplina, en la educación en enfermeria se privilegia los saberes de la práctica. Mientras el desarrollo de la disciplina se orienta hacia la utilización de teorías y modelos de enfermería, el currículo se caracteriza por continuar basándose predominantemente en el modelo biomédico 26-30. y la práctica bajo una orientación tecnicista ${ }^{27}$, descontextualizada, dadas desde métodos, tiempos, espacios, forma y contenido preestablecidos; esto es, se desconocen los procesos de significación del territorio y sus habitantes, los saberes propios, las experiencias y la contextualización del conocimiento; lo anterior plantea una dicotomía entre el discurso y la acción, entre la teoría y la praxis. 
En algunos textos ${ }^{27}$, el curriculum de enfermería, se describe como fundamentado desde una racionalidad científico-técnica, dada en primer lugar por las ciencias básicas y a continuación las prácticas clínicas. Lo anterior lleva a una visión instrumental en las relaciones entre teoría, práctica e investigación. Adicionalmente, la organización jerárquica del mismo, donde se presentan en primer lugar las ciencias básicas biológicas (anatomía, fisiología, bioquímica) y las psicosociales (psicología) así como los fundamentos de la ciencia enfermería (modelos y teorías) los cuales posteriormente se aplican en las diferentes asignaturas, dejando en última instancia las prácticas en los servicios, donde se aplican los conocimientos básicos a las situaciones de cuidado, contribuyen a esta escisión ${ }^{27}$.

En relación a la situación especial de la enseñanza de enfermería, los autores ${ }^{14}$. expresan que la metáfora de una laguna entre la teoría y la práctica está establecida, aduciendo que para algunos autores ella siempre existirá; pues se tiene diferentes tipos de conocimientos involucrados. Por otra parte, algunos profesores consideran que esta dicotomía se da, debido a que los conceptos teóricos se encuentran fuera de época, sugiriendo que la teoría requiere una reconceptualización y ante esto, hacen un llamado a que los profesores muestren lo relevante en la integración de estos diferentes conocimientos a los estudiantes:

Se describe en algunas publicaciones la presencia de una descontextualización del currículo con respecto a la realidad social y particular de cada región ${ }^{29}$. Ante esto, los autores consideran que la docencia en enfermería debe realizarse basada en los cambios vertiginosos de la sociedad y las necesidades de cada región específica, ante lo cual, se hace un llamado a la académica a realizar un cambio a nivel curricular, adaptando la teoría, los programas y los proyectos educativos a las necesidades particulares del contexto social ${ }^{29}$. Se resalta que el desarrollo disciplinar debe constituirse en un plan de gobierno ${ }^{31}$; en corresponsabilidad con los cambios del entorno, las modificaciones de la políticas públicas tanto en salud como en educación; esta responsabilidad debe asumirla la academia a través del acercamiento entre la teoría y la praxis, entre los discursos del aula y el cuidado de enfermería en su realidad contextual ${ }^{32-35}$.

Otra de las situaciones identificadas es la falta de articulación e integración entre la docencia-asistencia e investigación; la vinculación efectiva entre las instituciones de educación con los servicios y de estos con la investigación que es realizada en enfermería es de vital importancia. Lo anterior, es considerado como una fórmula para disminuir el divorcio entre la teoría y la práctica. En la revisión se encontró además que en muchos casos, los profesores de teoría y los de práctica actúan y enseñan desde perspectivas y discursos distantes; ${ }^{36,37}$. debido especialmente a la falta de articulación entre los procesos formativos de los profesionales con relación al cuidado frente a las estrategias de la práctica, que están más centradas en el hacer, la cual se gana a través de la experiencia directa frente a una situación. Lo anterior, de acuerdo con los textos revisados, lleva a una dicotomía entre la formación ofrecida por los profesores y las actividades realizadas en los servicios por los estudiantes y los enfermeros asistenciales ${ }^{39}$.

\section{Tendencia 02: estudios relacionados con los estudiantes}

Siete documentos aportan a la problematización de la relación entre teoría y praxis por parte de los estudiantes, correspondientes a un porcentaje de $20 \%$. Los resultados de las investigaciones indican que los estudiantes perciben una diferencia 
entre dos formas de conocimientos: el conocimiento académico (teórico) y el conocimiento en la práctica. Los textos muestran que el conocimiento académico se caracteriza por ser abstracto, acontextual, estable y rígido. Mientras que el conocimiento en la práctica es concreto y se halla en un proceso constante de comprensión-transformación- comprensión, es inestable, complejo, incierto y saturado de valores; refleja la vida real y el contexto del cual forman parte las personas ${ }^{45}$. Identificándose autores ${ }^{46}$ que definen el conocimiento teórico (saber teórico), como aquel que le permite al individuo establecer relaciones para comprender los sucesos y el conocimiento en la práctica (saber práctico), como el saber producto de una situación, en la cual se comparten habilidades, hábitos y conocimientos.

Se evidencia en los hallazgos que este saber es poco frecuente en los libros, pero sí descrito en las experiencias laborales de los enfermeros asistenciales, las cuales no puede ser formalizadas ni enseñadas por parte de los profesores a los estudiantes a partir de la teoría en el aula, puesto que es muy difícil hacerlo explícito verbalmente y sólo puede ser demostrado ${ }^{46}$. Evidenciando una diferencia entre dos formas de enseñanza: una forma de enseñanza para el saber teórico (enseñanza de la teoría) y otra forma particular de enseñanza de la práctica.

La falta de articulación entre los conocimientos adquiridos por los estudiantes durante su formación universitaria frente al quehacer de la práctica profesional, está dada especialmente por la racionalidad técnica, que entiende la práctica profesional como aquella en la cual la solución de los problemas se basa esencialmente en los conocimientos teóricos, así como una práctica centrada en la técnica y en los aparatos tecnológicos, que impide visibilizar el cuidado humano ${ }^{46}$. Adicionalmente, se enuncian otros factores que contribuyen a esta falta de articulación y que son descritos por los estudiantes: el dominio del modelo biomédico, la estructura rígida de las organizaciones, la imposición de otros modelos y la prevalencia en el cuidado de los aspectos físicos.

Vale la pena anotar que a pesar de los esfuerzos que realizan los profesores para desarrollar una docencia reflexiva y crítica, la realidad evidencia que mientras no exista una transferencia del conocimiento teórico de las aulas a la realidad de la práctica, será difícil conseguir la integración de los modelos enfermeros en el cuidado de las personas y por ende la relación de la teoría con la praxis enfermera.

\section{Tendencia 03: estudios relacionados con los enfermeros asistenciales}

Los resultados de diez investigaciones revisadas, correspondientes a un $29 \%$, expresan por parte de los profesionales su experiencia frente a la problematización de la relación teoría-praxis, la cual se centra en la identificación de una falta de aplicabilidad de los modelos teóricos en la práctica 47,48,49,51. Los enfermeros consideran que los modelos y teorías de enfermería se relegan de la práctica enfermera por su complejidad; aduciendo que los documentos de registros de enfermería favorecen poco trabajar bajo un modelo, posiblemente porque se han quedado obsoletos. Adicionalmente los estudios identificaron aspectos como la falta de dominio para su aplicación, pues muchos de ellos y ellas no fueron formados bajo el concepto del modelo enfermero, quizás debido a que los modelos conceptuales no se incluyen en el currículo de pregrado hasta mediados de los años setenta (en diferente intensidad y progresión según la escuela). Esto provoca que haya toda una generación de enfermeros asistenciales, que no está adecuadamente formada en este aspecto $^{51}$. 
Así mismo, los estudios evidencian la existencia de una diferencia entre la realidad y "Io que debería ser" ${ }^{\text {"7 }}$; los modelos de enfermería son considerados por los enfermeros muy a menudo difíciles de llevar a la práctica, espacio donde los profesionales están interesados en "lo que es", aunado a una preocupación por su habilidad técnica, considerando su utilización como importante desde un punto de vista teórico, fundamental para la construcción disciplinar, diferenciador respecto de otras profesiones y clarificador del rol profesional, pero obviado en la práctica asistencial.

Adicionalmente, se considera que existen factores que dificultan la relación entre teoría y praxis, los cuales fueron identificados por los enfermeros asistenciales, como: limitaciones debidas a la ideología médica y biologicista dominante ${ }^{47}$, la estructura organizativa de las instituciones, la elevada presión asistencial en las unidades de trabajo, la propia complejidad de los modelos y la existencia de reglas encubiertas por las que son socializados los profesionales según las necesidades de las instituciones de forma que prevalece el cuidado de aspectos físicos sobre el resto $^{47}$, así como el hecho de que durante mucho tiempo haya prevalecido una visión biologicista y médica sobre todo lo relacionado con la salud, la enfermedad y la persona que vive diferentes circunstancias por estos estados. Adicionalmente, algunos estudios encontraron resistencia al cambio y la percepción de falta de aplicabilidad de los modelos teóricos en el trabajo diario, llegando a la conclusión, en algunos estudios de que los profesionales no se sienten satisfechos con la relación que hay entre lo que aprendieron durante su formación y lo que aplican en su práctica diaria $^{47,48,55}$.

\section{DISCUSIÓN}

La revisión integradora de literatura es un tipo de investigación que posibilita una revisión amplia referente a las revisiones; permite la inclusión de estudios experimentales y no experimentales que posibilitan la comprensión completa del fenómeno analizado, combina datos de la literatura teórica y empírica ${ }^{56,57}$ de tal forma que facilita que sean evidenciadas las lagunas de conocimiento existentes y formular conclusiones de un tema especifico ${ }^{58}$; en este caso particular, frente a la problematización de la relación de la teoría con la praxis en enfermería.

A partir de los datos encontrados, se constató la existencia de una dicotomía permanente tanto en la práctica educativa por parte de los profesores, en la formación de los estudiantes y en la práctica asistencial. Esta dicotomía está dada por parte de los profesores, entre el discurso y la acción, entre la teoría y la praxis ${ }^{23,31,36,37 .}$.

En los estudiantes, se reconoce a través de la diferenciación entre dos formas de conocimientos: el conocimiento académico (teórico) y el conocimiento en la práctica, que están generados por dos formas diferentes de enseñanza. $Y$ en los enfermeros asistenciales, se evidencia en la realidad de lo que "debería ser" en la cual están fundamentados los modelos de enfermería, frente a "lo que es", que caracteriza la práctica asistencial.

A partir del análisis de los estudios revisados se identificó a la falta de consenso entre los aspectos relacionados con la teoría (ciencias de enfermería) y los aspectos relacionados con la práctica enfermera (práctica profesional), como uno de los principales factores asociados a la problematización de relación entre la teoría y la praxis, por parte de los diferentes actores; mientras el desarrollo de la disciplina se orienta hacia la utilización de teorías y modelos de enfermería, el currículo se 
caracteriza por continuar basándose predominantemente en el modelo biomédico ${ }^{27-}$ 30,34. y la práctica está dada principalmente bajo una orientación tecnicista ${ }^{27,34}$. Adicionalmente, se describen otros factores como el dominio del modelo biomédico, la estructura organizativa de las instituciones, la elevada presión asistencial en las unidades de trabajo, así como el hecho que durante mucho tiempo haya prevalecido una visión biologicista y médica sobre todo lo relacionado con la salud y la enfermedad.

Cabe destacar como uno de los factores que ha contribuido al establecimiento de esta problemática y es descrito en numerosas publicaciones, la presencia de una descontextualización del currículo con respecto a la realidad política, social y particular de cada región ${ }^{29,35}$. Ante lo cual, se hace un llamado a la academia a realizar un cambio a nivel curricular, adaptando la teoría, los programas y los proyectos educativos a las necesidades particulares del contexto social. Se resalta cómo algunos autores consideran que el desarrollo disciplinar debe constituirse en un plan de gobierno ${ }^{31}$ que acoja los cambios del entorno, las modificaciones de la políticas públicas tanto en salud como en educación y esta responsabilidad debe asumirla la academia a través del acercamiento entre la teoría y la praxis, entre los discursos del aula y el cuidado en su realidad contextual.

A partir del análisis realizado a través del estado del arte, se considera la teoría y la praxis como variables dicotómicas--difíciles de unir, ya que constituyen mundos amplios e intrínsecamente complejos. Sin embargo, de su relación, depende la coherencia de lo que se enseña, del cuidado de enfermería, así como de la identidad y desarrollo profesional. Por esta razón, se hace necesario acercar las relaciones entre estos dos mundos a partir del reconocimiento de esta realidad. El conocimiento respecto de los aspectos que constituyen la problematización de la relación entre ellas es fundamental, buscando establecer alguna forma de relación que propenda por brindar claridad y unificar los conceptos a fin de contribuir desde la docencia al reconocimiento y fortalecimiento de la profesión.

\section{CONCLUSIONES}

La complejidad de la relación entre la teoría y la praxis es evidente. La literatura muestra que mientras no exista una transferencia del conocimiento teórico de las aulas a la realidad de la práctica, será difícil conseguir la integración de la teoría con la praxis enfermera.

Entre las limitaciones del estudio, se destaca la escasa bibliografía identificada, más teniendo en cuenta la importancia del tema y la preocupación constante que genera tanto en los profesores, estudiantes, como en los enfermeros asistenciales. Sin embargo, es de resaltar como se encontró un mayor número de estudios centrados en el papel de los profesores, lo cual da a entender el protagonismo de ellos frente al problema y la posibilidad desde la docencia de buscar un acercamiento. Es Ilamativo, cómo los estudios no se centran en investigar y ampliar más respecto a las causas de este distanciamiento; la mayoría son estudios descriptivos que se centran en describir el problema y cómo este se percibe por parte de los sujetos inmersos en él.

En general, los resultados indican la necesidad de realizar investigaciones con diferentes perspectivas, que aborden otras situaciones que pueden incidir en la problemática descrita; se sugiere la realización de más estudios desarrollados con diferentes metodologías a fin de ampliar la comprensión de la problemática descrita y 
su incidencia en el desempeño de los profesionales. Se sugiere adicionalmente, considerar el contexto específico y particular de las diferentes áreas de desempeño profesional a fin de ampliar la percepción frente a esta temática y aportar contribuciones que permitan mejorar la formación de profesionales de enfermeria articulando aspectos teóricos, técnicos, políticos, socioculturales, éticos y estéticos, que constituyen la praxis enfermera

\section{REFERENCIAS}

1. Bondarenko Pisemskaya N, El concepto de teoría: de las teorías intradisciplinarias a las transdisciplinarias. Revista de Teoría y Didáctica de las Ciencias Sociales 2009 461-477. Disponible en: http://www.redalyc.org/articulo.oa?id=65213215010 . Fecha de consulta: 26 de septiembre de 2016.

2. E., \& LUIS, J. Diccionario de la Lengua Española vol. I. Madrid: Calpe; 2002

3. Bárcena, F. La experiencia reflexiva en educación. Barcelona: Paidós; 2005.

4. Rivera, L. N. Saber y experiencia de el/la estudiante de enfermería en sus prácticas de cuidado - Tesis Doctoral. Universidad de Barcelona. 2013.

5. Medina, J. L. La pedagogía del cuidado: saberes y prácticas en la formación universitaria en enfermería. Barcelona: Laertes;1999.

6. Acebedo, S. Narrativa y conocimiento práctico. Experiencias y prácticas de las enfermeras "expertas" en uci. Once relatos y veintitantas historias - Tesis Doctoral. Universitat Rovira. 2012.

7. Bourdieu, P. El sentido práctico. Buenos Aires: Siglo XXI editores;2007.

8. Schön, D. El profesional reflexivo: cómo piensan los profesionales cuando actúan. Barcelona: Paidós;1998.

9. Toulmin, S. Regreso a la razón. Barcelona: Ediciones Península;2003.

10. Benner, P. Links between philosophy, theory, practice, and research. Can J Nurs Res. 2000; 32(2): 7-13.

11. Medina, J. Deseo de cuidar y voluntad de poder. Barcelona: Universitat de Barcelona; 2005

12. López, R., \& Torres, M. Praxis en enfermería: implicaciones. Enfermería Universitaria 2009; 6(4): 36-38.

13. Durán de Villalobos, M. M. Marco epistemológico de la enfermería. Aquichán [serial on the Internet]. 2002 Dec [cited 2016 Mar 14] ; 2( 1 ): 7-18. Available from: http://www.scielo.org.co/scielo.php?script=sci_arttext\&pid=S1657-

$59972002000100003 \&$ Ing=en.

14. Medina, E. La formación de pregrado y la realidad asistencial en la profesión enfermera - Tesis Doctoral. Universidad de Alicante.2013

15. Hernández, J., Moral, P. y Esteban- Albert, M. Fundamentos de la enfermeria. Teoría y método. Madrid: McGraw-Hill Interamericana; 2003.

16. Collière, M. Promover la vida. Madrid, España: McGraw-Hill;1993.

17. Durán de Villalobos, M. M. La teoría, soporte de la ciencia y práctica de enfermería: tendencias. av.enferm. [serial on the Internet]. 2012 Jan [cited 2015 Oct 10] $\quad$; 30( $1 \quad$ ): 9-12. Available from: http://www.scielo.org.co/scielo.php?script=sci_arttext\&pid=S0121-

45002012000100001\&lng=en

18. ACOFAEN. Directrices para la enseñanza de enfermería en la educación superior. Bogotá: Asociación Colombiana de Facultades de Enfermería. 2006.

19. Scherer Zeyne Alves Pires, Scherer Edson Arthur. Reflexiones sobre la enseñanza de enfermería en la postmodernidad y la metáfora de una laguna teóricopráctica. Rev. Latino-Am. Enfermagem [Internet]. 2007 June [cited 2015 Nov 05] ; 
http://www.scielo.br/scielo.php?script=sci_arttext\&pid=S0104-

11692007000300021 \&lng=en.http://dx.doi.org/10.1590/S0104-1692007000300021.

20. Benner, P., Sutphen, M., Leonard, V., \& Day, LEducating nurses: a call for radical transformation. The Carnegie Foundation for the Advancement of Teaching; 2009.

21. Ganong LH. Integrative reviews of nursing research. Res Nurs Health. 1987;10(1):1-11.

22. ACOFAEN. Directrices para la enseñanza de enfermería en la educación superior. Bogotá: Asociación Colombiana de Facultades de Enfermería. 2006.

23. ACOFAEN. PLAN DE DESARROLLO 2009 - 2018.Bogotá: Asociación Colombiana de Facultades de Enfermería.2009.

24. Durán de Villalobos MM. Teoría de enfermería ¿un camino de herradura?. Aquichan 2007 161-173. Disponible en: http://redalyc.org/articulo.oa?id=74107207

25. Durán MM. Enseñanza de la disciplina de enfermería. ALADEFE y Universidad de Antioquia. VII Conferencia Iberoamericana de Educación en Enfermería: Necesidades, Tendencias y Desafíos. Medellín 6-8 de octubre de 2006. Bogotá: Biblioteca Las Casas; 2006; 1-5.Disponible en http://www.indexf.com/lascasas/documentos/lc0156.ph

26. Medina, J.L, Sandín, E. La complejidad del cuidado y el cuidado de la complejidad: un tránsito pedagógico de los reduccionismos fragmentantes a las lógicas no líneales de la complejidad. Texto contexto - enferm. [Internet]. 2006 June [cited 2015 Oct 15] ; 15( 2 ): 312-319. Available from: http://www.scielo.br/scielo.php?script=sci_arttext\&pid=S0104-

07072006000200016\&Ing=enhttp://dx.doi.org/10.1590/S01047072006000200016 27. Moya José Luis Medina, Prado Marta Lenise do. El curriculum de enfermería como prototipo de tejné: racionalidad instrumental y tecnológica. Texto contexto enferm. [Internet]. 2009 Dec [cited 2015 Oct 15] ; 18( 4 ): 617-626. Available from: http://www.scielo.br/scielo.php?script=sci_arttext\&pid=S0104-

07072009000400002\&lng=en.http://dx.doi.org/10.1590/S0104-07072009000400002 28. Moya Jose Luis Medina, Backes Vânia Marli Schubert, Prado Marta Lenise do, Sandin Mari Paz. La enfermería como grupo oprimido: las voces de las protagonistas. Texto contexto - enferm. [Internet]. 2010 Dec [cited 2015 Oct 15] ; 19( 4 ): 609617. Available from: http://www.scielo.br/scielo.php?script=sci_arttext\&pid=S0104$07072010000400002 \&$ Ing=en.http://dx.doi.org/10.1590/S0104-07072010000400002.

29. Mejías, M. Modelo de enseñanza para enfermería. Tesis Doctoral. Universidad de Carabobo; 2013.

30. Moreno Fergusson M, E. Importancia de los modelos conceptuales y teorías de enfermería: experiencia de la Facultad de Enfermería de la Universidad de La Sabana. Aquichán [serial on the Internet]. 2005 Oct [cited 2015 Oct 30] ; 5( 1 ): 4455. Available from: http://www.scielo.org.co/scielo.php?script=sci_arttext\&pid=S165759972005000100005\&lng=en.

31. Argote, L. Á., Burbano, C., Santamaría, C., \& Vásquez, M. L. Disciplinary development: the reason for being, sensing, thinking and doing in nursing. Colombia Médica 2011;

http://colombiamedica.univalle.edu.co/index.php/comedica/article/view/823/1561

(ultimo acceso 27 de septiembre de 2016).

32. Bastidas, C. V., Bonilla, C. P. Olivella, M. La formación disciplinar en programas de pregrado en enfermería: la experiencia de la universidad del Tolima-Colombia. Enfermería Docente, 2011; 95: 34-37.

33 Guillén, M. Teorías aplicables al proceso de atención de enfermería en Educación Superior. Rev Cubana Enfermer [revista en la Internet]. 1999 Abr [citado 2015 Nov 05] 15(1): 10-16.

Disponible en: 
http://scielo.sld.cu/scielo.php?script=sci_arttext\&pid=S0864-

03191999000100002\&lng=es

34. Pinto, J, \& Pepe A. La formación del enfermero: contradicciones y desafíos de la práctica pedagógica. Rev. Latino-Am. Enfermagem [Internet]. 2007 Feb [cited 2015 Nov 05] ; 15( 1 ): 120-126. Available from: http://www.scielo.br/scielo.php?script=sci arttext\&pid=S0104-

$11692007000100018 \&$ Ing=en.http://dx.doi.org/10.1590/S0104-11692007000100018.

35. Bernal, M. Ponce, G. Propuesta para la enseñanza del cuidado en enfermería. Revista Enfermería Universitaria ENEO-UNAM, 2009 ; 6(6): 33-41.

36 Tejada Tayabas LM, Castro-Ramírez J, Miranda-Herrera MM, Acosta Ramírez L, Alcántara Hernández E. Evaluación cualitativa de un programa de formación de enfermeras. La perspectiva de los profesores y los estudiantes. Investigación y Educación en Enfermería 2008XXVI80-88. Disponible en: http://55mmm.redalyc.org/articulo.oa? $\mathrm{id}=105215278006$. Fecha de consulta: 5 de noviembre de 2015.

37. Mantilla-Pastrana M. I., Gutiérrez-Agudelo M. C.. Procesos de evaluación del aprendizaje del cuidado en la práctica de estudiantes de Enfermería. Enferm. univ [revista en la Internet]. 2013 Abr [citado 2015 Nov 05] ; 10( 2 ): 43-49. Disponible en:

http://www.scielo.org.mx/scielo.php?script=sci_arttext\&pid=S1665$70632013000200003 \&$ Ing=es.

38.Scherer Z , Scherer. Reflexiones sobre la enseñanza de enfermería en la postmodernidad y la metáfora de una laguna teórico-práctica. Rev. Latino-Am. Enfermagem [Internet]. 2007 June [cited 2015 Nov 05] ; 15( 3 ): 498-501. Available from:

http://www.scielo.br/scielo.php?script=sci_arttext\&pid=S0104$11692007000300021 \&$ lng=enhttp://dx.doi.org/10.1590/S0104-11692007000300021.

39 Palencia, E. Reflexión sobre el ejercicio docente de enfermería en nuestros días. Investigación y Educación en Enfermería, 2006; 24(2):130-134. Retrieved November 06, 2015, from http://www.scielo.org.co/scielo.php?script=sci_arttext\&pid=S0120$53072006000200014 \& \operatorname{lng}=$ en\&tlng=es

40. Mejía Lopera, M. E. Aplicación de algunas Teorías de Enfermería en la Práctica Clínica. Index Enferm [revista en la Internet]. 2008 Sep [citado 2015 Nov 05] ; 17(3): 197-200. Disponible en: http://scielo.isciii.es/scielo.php?script=sci_arttext\&pid=S1132$12962008000300010 \&$ Ing=es.

41 Santos-Ruiz S, López-Parra M, Sánchez-Rueda G, Bardallo-Porras L, FernándezDonaire L, Zabalegui-Yárnoz A et al. Modelos teóricos y estudiantes de enfermería: aspectos motivacionales para su elección durante las prácticas clínicas. Educ. méd. [revista en la Internet]. 2011 Jun [citado 2015 Nov 05] ; 14(2): 119-127. Disponible en:

$18132011000200008 \& \operatorname{lng}=$ es.

http://scielo.isciii.es/scielo.php?script=sci_arttext\&pid=S1575-

42. Krederdt, S. L. Reflexiones sobre el cuidar-cuidado de enfermería: estudio etnográfico en enfermeras docentes de la Universidad Nacional Mayor de San Marcos. Tesis Doctoral. Universidad Nacional Mayor de San Marcos. 2006.

43. Castillo, S. Práctica pedagógica de los formadores expertos en la formación clínica de estudiantes de enfermería - Tesis Doctoral. Universidad de Barcelona; 2014.

44. Rivera L. N. Saber y experiencia de el/la estudiante de enfermería en sus prácticas de cuidado. Tesis Doctoral. Universidad de Barcelona. 2013.

45 Molina P., Jara P. El saber práctico en Enfermería. Rev Cubana Enfermer [revista en la Internet]. 2010 Jun [citado 2015 Oct 13] ; 26(2): . Disponible en: http://scielo.sld.cu/scielo.php?script=sci_arttext\&pid=S0864-

03192010000200005\&lng=es. 
46 Medina, J. L., \& Castillo, S. La enseñanza de la enfermería como una práctica reflexiva. Texto contexto - enferm. [Internet]. 2006 June [cited 2015 Oct 14] ; 15( 2 ): 303-311. Available from: http://www.scielo.br/scielo.php?script=sci_arttext\&pid=S0104-

07072006000200015\&lng=en.http://dx.doi.org/10.1590/S0104-07072006000200015

47. Santos-Ruiz S, López-Parra M, Várez-Peláez S, Abril-Sabater D. Perspectiva de la enfermería de un centro sociosanitario sobre la aplicabilidad y utilidad de los modelos enfermeros en la práctica. Gerokomos [revista en la Internet]. 2008 Dic [citado 2015 Oct 13] ; 19(4): 32-37. Disponible en: http://scielo.isciii.es/scielo.php?script=sci_arttext\&pid=S1134-

928X2008000400004\&lng=es

48. Santos Ruiz S, López Parra M, Varez Peláez S, Abril Sabater D. Propuesta de un modelo teórico para la práctica enfermera. NURE Inv. [Revista en Internet] 2010 EneFeb. [fecha acceso]; 7 (44): Disponible en:

http://www.fuden.es/FICHEROS_ADMINISTRADOR/ORIGINAL/promodenf.pdf 49. López-Parra M, Santos-Ruiz S, Varez-Peláez S, Abril-Sabater D, Rocabert-Luque M, Ruiz-Muñoz M et al. Reflexiones acerca del uso y utilidad de los modelos y teorías de enfermería en la práctica asistencial. Enferm Clin 2006; 16(4): 218-21.

50. Reyes, J. Jara, P. Merino, J. Adherencia de las enfermeras/os a utilizar un modelo teórico como base de la valoración de enfermería. Cienc. enferm. [Internet]. 2007 Jun [citado 2015 Nov 09] ; 13( 1 ): 45-57. Disponible en: http://www.scielo.cl/scielo.php?script=sci_arttext\&pid=S0717-

95532007000100006\&lng=eshttp://dx.doi.org/10.4067/S0717-95532007000100006.

51. Rodríguez, R. Modelos de enfermería integrados al cuidado del paciente nefrológico en el contexto cubano. Rev Cubana Enfermer [revista en la Internet]. 2012 Dic [citado 2015 Nov 09] ; 28(4): 474-484. Disponible en: http://scielo.sld.cu/scielo.php?script=sci_arttext\&pid=S0864-

03192012000400004\&lng=es.

52. Triviño, Z., \& Sanhueza, O. Teorías y modelos relacionados con calidad de vida en cáncer y enfermería. Aquichán 2005; 5(1): 20-31.

53. Acebedo, S. Narrativa y conocimiento práctico. Experiencias y prácticas de las enfermeras "expertas" en uci. Once relatos y veintitantas historias - Tesis Doctoral. Universitat Rovira. 2012.

54. Zapico, F. Avances en la práctica de la profesión de enfermería. Visiones de una misma realidad - Tesis Doctoral. Universitat de Barcelona. 2005.

55. Francisco del Rey, J. C. De la práctica de la enfermería a la teoría enfermera: Concepciones presentes en el ejercicio profesional .Tesis Doctoral. Universidad de Alcalá; 2008.

56. Whittemore $\mathrm{R}$, Knafl K. The integrative review: update methodology. J Adv Nurs. 2005;52(5):546-53.

57. Souza MT, Silva MD, Carvalho R. Revisão integrativa: o que é e como fazer. Rev. Einstein, 2010; 8(1 Pt 1):102-6.

58. Guirao-Goris JA, Olmedo Salas A, Ferrer Ferrandis E. El artículo de revisión. Rev. Iberoam. Enfermería Comunitaria [Internet]. 2008;1(1):6. [visitado 2012 enero 10]. Disponible en: http://revista.enfermeriacomunitaria.org/articuloCompleto

ISSN 1695-6141

(c) COPYRIGHT Servicio de Publicaciones - Universidad de Murcia 